\title{
SEMICELLULARITY, DECOMPOSITIONS AND MAPPINGS IN MANIFOLDS
}

BY

\section{DONALD CORAM(1)}

\begin{abstract}
If $X$ is an arbitrary compact set in a manifold, we give algebraic criteria on $X$ and on its embedding to determine that $X$ has an arbitrarily small, closed neighborhood each component of which is a p-connected, piecewise linear manifold which collapses to a $q$-dimen sional subpolyhedron from some $p$ and $q$. This property generalizes cellularity. The criteria are in terms of $U V$ properties and Alexander-Spanier cohomology. These criteria are then applied to decide when the components of a given compact set in a manifold are elements of a decomposition such that the quotient space is the n-sphere. Conversely, algebraic criteria are given for the point inverses of a map between manifolds to have arbitrarily small neighborhoods of the type mentioned above; these criteria are considerably weaker than for an arbitrary compact set.
\end{abstract}

1. Introduction. The concept of cellularity, introduced by M. Brown [7], has been important to the study of embeddings of compact sets in manifolds. This paper deals with the following more general property. We say that a compact set $X$ in a manifold is $(p, q)$-semicellular if $X$ has an arbitrarily small closed neighborhood each component of which is a $p$-connected piecewise linear manifold which collapses to a $q$-dimensional subpolyhedron. In $\$ \$ 1-5$, we give algebraic criteria for semicellularity analogous to D. R. McMillan's criterion for cellularity [19]. The latter part of this paper presents applications of these results to questions about extending decompositions and about mappings on manifolds.

The problem of extending decompositions was first raised by R. H. Bing [ 5 ] and R. J. Bean [4]. It asks: Given a decomposition $G$ of $S^{n}$ (the $n$-sphere), under what conditions is there a "nice" (in some precise sense) decomposition $H$ of $S^{n}$ such that each nondegenerate element of $G$ is an element of $H$ and $S^{n} / H \cong S^{n}$.

Presented to the Society, April 21, 1971 under the title Semicellularity in manifolds; received by the editors March 17, 1972 and, in revised form, July 24, 1973.

AMS (MOS) subject classifications (1970). Primary 57 A60; Secondary 54B15, 54C10, $57 \mathrm{A15}$.

Key words and phrases. Neighborhoods of compacta, UV properties, extending decompositions, mappings on manifolds.

(1) This paper results from the author's $\mathrm{Ph}$.D. thesis written under Professor $\mathrm{D}$. R. McMillan, Jr., at the University of Wiscon sin, Madison. 
There are variations of this question for manifolds other than $S^{n}$, too. Bing and Bean answer the question where $n=3$ and "nice" means monotone upper semicontinuous. B. J. Ball and R. B. Sher [3] have considered this question where $n \neq 4$ and "nice" means cell-like. This paper considers the question in higher dimensions where "nice" means $U V^{p}$. (The terms used above are defined later.)

The converse of this problem is also studied. The result could be called a semicellularity criterion for maps analogous to R. C. Lacher's cellularity criterion for maps [16]. It gives geometric conclusions for the point inverses of a map between manifolds given algebraic conditions on them.

The term manifold as used here allows nonempty boundary. If $M$ is a manifold, Int $M$ denotes the interior of $M$ (those points with a neighborhood homeomorphic to Euclidean space); and $\mathrm{Bd} M$ denotes the boundary of $M$ (those points with a neighborhood homeomorphic to Euclidean half-space and without a neighborhood homeomorphic to Euclidean space). If $A \subset B, \mathrm{Cl} A$ denotes the closure of $A$ in $B$. A $q$-spine for a triangulated manifold is a $q$-dimensional subpolyhedron to which the manifold collapses. The standard $n$-ball and $n$-sphere are denoted $B^{n}$ and $S^{n}$, respectively.

For an arbitrary compact set $X$ in a manifold, we use "UV properties" in place of the usual homeotopy-connectedness. We say that $X$ has property $U V^{k}$ provided given a neighborhood $U$ of $X$, there is a neighborhood $V$ of $X$ in $U$ such that each singular $i$-sphere $(i \leq k)$ in $V$ is null-homotopic in $U$. Also $X$ is cell-like, or $X$ has property $U V^{\infty}$, provided given a neighborhood $U$ of $X$, there is a neighborhood $V$ of $X$ in $U$ such that $V$ is contractible in $U$. Both of these properties are independent of the particular embedding of $X$ in manifold [1]. One property which definitely depends on the embedding is property CC: Given a neighborhood $U$ of $X$, there is a neighborhood $V$ of $X$ in $U$ such that each loop in $V-X$ is null-homotopic in $U-X$. For cohomology we use Alexander-Spanier cohomology with integral coefficients [23] denoted $\bar{H}^{*}(X)$. To denote ordinary (singular, integral) homology and cohomology, we drop the bar over the $H$.

The main theorem is the following.

Theorem 1. Let $X$ be a compact proper subset of the interior of a piecewiselinear m-manifold $M$. If $X$ bas $C C$, eacb component of $X$ bas $U V^{p}$, and $\bar{H}^{i}(X)=0$ for $i \geq q+1$, then $X$ bas an arbitratily small compact neighborbood eacb component of which is a p-connected polybedral m-manifold that bas a q-spine provided $p, q$ and $m$ satisfy one of the following inequalities:

(a) $p=1, q \geq 2$ and $m \geq 5$;

(b) $2 \leq p \leq q-1 \leq m-5$;

(c) $p=2$ and $q=m-3 \geq 3$;

(d) $p>q-1, p \geq 1$ and $m \geq 5$. 
The division into cases of the inequalities is determined by the proof. The cases are mutually exclusive. In case (d) the conclusion is that each component of the neighborhood is an $m$-cell. The converse of the theorem is mostly true. The conclusion does imply that each component of $X$ has $U V^{p}$ and $\bar{H}^{i}(X)=0$ for $i \geq q+1$, but $X$ does not necessarily have CC unless $q \leq m-3$.

2. The proof under inequality (a). Given any neighborhood $U$ of $X$, there is a compact neighborhood $N$ of $X$ in $U$ each component of which is a polyhedral $m$-manifold. Since $N$ has only finitely many components and the argument proceeds one component at a time, we will, for notational simplicity, assume that $N$ is connected. By choosing $N$ so that each loop in $N-X$, and specifically in Bd $N$, is null-homotopic in $U-X$ and using a standard surgery argument on $\mathrm{Bd} N$ (see [8], [6] and [10]), we may also assume that $\mathrm{Bd} N$ is simply connected. Furthermore, Int $N-X$ is simply connected since any loop there bounds a singular disk in $U-X$ which can be "capped off at the boundary". Similarly by choosing $N$ so that each loop in $N$ is null-homotopic in $U$, we may assume $N$ is simply connected. Now, if $q \geq m-1$, we are done; so henceforth assume $q \leq m-2$.

By duality

$$
H_{m-i}(\text { Int } N, \text { Int } N-X)=\bar{H}^{i}(X)=0 \text { for } i \geq q+1,
$$

and hence

$$
H_{j}(\text { Int } N \text {, Int } N-X)=0 \text { for } 0 \leq j \leq m-q-1 \text {. }
$$

Since $H_{1}($ Int $N$, Int $N-X)=0$ implies that Int $N-X$ is 0 -connected, both Int $N$ and Int $N-X$ are 1-connected by the first paragraph. Consequently, (Int $N$, Int $N-X)$ is $(m-q-1)$-connected by the Hurewicz theorem.

Now $m-q-1 \leq m-3$, so we can apply Stallings engulfing theorem [24] to Int $N$ in order to obtain a piecewise-linear homeomorphism $b: N \rightarrow N$ such that $b$ is the identity in a neighborhood of $\mathrm{Bd} N$ and the $(m-q-1)$-skeleton $P$ of $N$ is contained in $b(N-X)$. Then $b^{-1}(P) \cap X=\varnothing$. Let $Q$ be the complementary skeleton to $P$ and $R=b^{-1}(Q)$. Then $X$ is contained in a regular neighborhood of $R$ [13, pp. 49-57], [25, L. 8.1]. This is the neighborhood desired since $\operatorname{dim} R=\operatorname{dim} Q=\operatorname{dim} N-\operatorname{dim} P-1=q$ and any loop in it bounds a singular disk $N$ which can be homotoped off $b^{-1}(P)$ by general position.

3. The proof under inequality (b). In this case we can apply case (a) to obtain an arbitrarily small neighborhood of $X$ each component of which is a 1 connected manifold with a $q$-spine. We can make each spine $p$-connected by coning over its $p$-skeleton, so the problem is to embed the cone in the neighborhood. The next two lemmas allow us to overcome this difficulty. 
Lemma 2. Let $f: Q \rightarrow N$ be a piecewise-linear, general position map from a polybedron $Q$ into a piecewise-linear manifold $N$ such that $\operatorname{dim} S(f) \leq r$ where $r \geq 0$, and $H_{k}(Q)=0$ for $1 \leq k \leq p$ where $p \geq 1$. Then $H_{k}(f(Q))=0$ for $r+2 \leq$ $k \leq p$.

Proof. Let $U$ be a second derived neighborhood of $S=S(f)$ in $Q$. Then $V=f(U)$ is a second derived neighborhood of $T=f(S)$ in $f(Q)$. Since $\operatorname{dim} S \leq r$ and $\operatorname{dim} T \leq r, H_{k}(U)=H_{k}(V)=0$ for $k \geq r+1$. Consider the following diagram where $r+2 \leq k \leq p$.

$$
\begin{aligned}
& \rightarrow H_{k}(U-S) \longrightarrow H_{k}(Q-S) \longrightarrow H_{k}(Q) \rightarrow H_{k-1}(U-S) \rightarrow \\
& \downarrow+1 \approx \quad \downarrow \\
& \rightarrow H_{k}(V-T) \rightarrow H_{k}(f(Q)-T) \rightarrow H_{k}(f(Q)) \rightarrow H_{k-1}(V-T) \rightarrow
\end{aligned}
$$

The horizontal rows come from Mayer-Vietoris sequences. The vertical arrows are induced by $f$. By the five lemma

$$
H_{k}(f(Q)) \cong H_{k}(Q)=0 \text { for } r+2 \leq k \leq p .
$$

Lemma 3. Let $R$ be a connected, q-dimensional polydron such that $H_{k}(R)=0$ for $r+2 \leq k \leq p$ where $0 \leq r \leq q-3$. Then there exists a q-dimensional, p-connected polybedron $Q \supset R$ such that $\operatorname{dim}(Q-R) \leq r+3$.

Proof. Let $P=R \cup C\left(R^{r+1}\right)$ be the polyhedron obtained from $R$ by attaching the cone over the $(r+1)$-skeleton of $R$. By the Mayer-Vietoris sequence we have

$$
H_{k}(R) \oplus H_{k}\left(C\left(R^{r+1}\right)\right) \rightarrow H_{k}(P) \stackrel{\partial^{*}}{\longrightarrow} H_{k-1}\left(R^{r+1}\right) .
$$

Because $H_{k}(R)=0$ for $r+2 \leq k \leq p$ and $C\left(R^{r+1}\right)$ is contractible, $\partial^{*}$ is injective for $r+2 \leq k \leq p$. For $k=r+2, H_{r+1}\left(R^{r+1}\right)$ is a free Abelian group of some rank, say n. For $k \geq r+3, H_{k-1}\left(R^{r+1}\right)=0$ and so $H_{k}(P)=0$ for $r+3 \leq k \leq p$.

Now, since $P$ is $(r+1)$-connected, $H_{r+2}(P) \cong \pi_{r+2}(P)$ and, therefore, there is a set of free generators for $H_{r+2}(P)$ such that each one. is a polyhedral singular $(r+2)$-sphere $g_{l}: S^{r+2} \rightarrow P$ for $l=1,2, \cdots, n$. Let $\left\{B_{1}, B_{2}, \cdots, B_{n}\right\}$ be a disjoint collection of $(r+3)$-balls, and denote their union by $B$. Let $S=\operatorname{Bd} B$ and define a map $g: S \rightarrow P$ by $g \mid B d B_{l}=g_{l}$. Then let $Q=P \cup_{g} B$ be the polyhedron obtained by attaching $B$ to $P$ by $g$.

We now claim that $Q$ is $p$-connected. Let $U$ be a second derived neighborhood of $S$ in $B$. Then $V=P \cup_{8} U$ is a second derived neighborhood of $P$ in $Q$. Since the point-set interiors of $\stackrel{g}{V}$ in $Q$ and of each $B_{l}$ in $Q$ are 1-connected and each intersection is 0 -connected, $Q$ is 1 -connected by Van Kampen's theorem and induction. 
Because of the Hurewicz theorem, we now need only to prove that $H_{k}(Q)=0$ for $2 \leq k \leq p$ and for this consider the following commutative diagram.

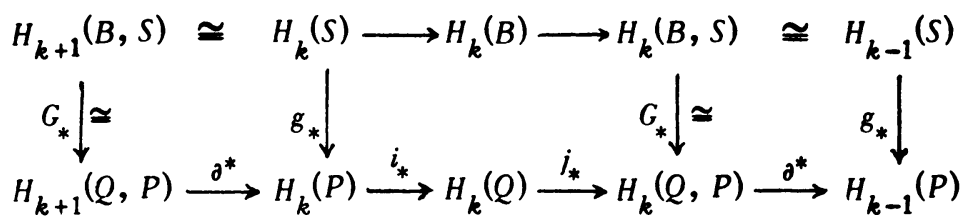

The isomorphisms in the top line occur because $H_{k}(B)=0$ for $k \geq 1$. The vertical arrows labeled $G_{*}$ are induced by the map $G:(B, S) \rightarrow(Q, P)$ defined as inclusion on Int $B$ and as $g: S \rightarrow P$ on $B d B=S$. It can be shown to induce isomorphisms in every dimension because the inclusion $B-S \subset Q-P$ is a homeomorphism. Then $H_{k}(Q, P) \cong H_{k-1}(S)=0$ for $k \geq 2$ and $k \neq r+3$. Therefore, $H_{k}(Q) \cong H_{k}(P)=0$ for $2 \leq k \leq p$ and $k \neq r+2, r+3$. For $k=r+2$, we have the short exact sequence

$$
0 \rightarrow \text { ker } i_{*} \rightarrow H_{r+2}(P) \stackrel{i_{*}}{\longrightarrow} H_{r+2}(Q) \stackrel{i_{*}}{\longrightarrow} 0 .
$$

But ker $i_{*}=\operatorname{im} \partial_{*}=\operatorname{im} g_{*}$ by exactness and the presence of isomorphisms on the

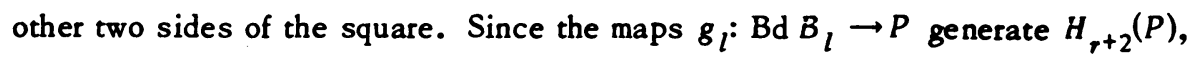
$g_{*}$ is surjective and $H_{r+2}(Q)=0$. For $k=r+3$, we have the short exact sequence

$$
0 \rightarrow H_{r+3}(P) \stackrel{i_{*}}{\longrightarrow} H_{r+3}(Q) \stackrel{i_{*}}{\longrightarrow} \text { im } j_{*} \rightarrow 0 \text {. }
$$

However, im $j_{*}=\operatorname{ker} \partial_{*} \cong \operatorname{ker} g_{*}$. Since the maps $g_{l}$ freely generate $H_{r+2}(P), g_{*}$ is injective, so im $j_{*}=0$. Thus, $H_{r+3}(Q) \cong H_{r+3}(P)=0$. In sum, $H_{k}(Q)=0$ for $1 \leq k \leq p$ which was what was needed to complete the proof of the lemma.

To prove Theorem 1 in case (b), let $n=\max \{p+q-m+3,1\}$ and choose neighborhoods $U_{n p}, U_{n p-1}, \cdots, U_{1}, U_{0}$ of $X$ by property $U V^{p}$ and case (a) such that $U_{0}$ is arbitrarily small, Int $U_{i}$ contains closure $\left(U_{i+1}\right)$, each singular $k$. sphere in $U_{i+1}$ is null-homotopic in $U_{i}$ for $1 \leq k \leq p$, and $U_{n p}$ is a compact neighborhood each component of which is a polyhedral $m$-manifold that has a $q$-spine. Let $R$ denote the union of these $q$-spines.

We will prove by induction that for $k=1,2, \cdots, n$, there is a polyhedron $Q_{k}$ of dimension $q$ each component of which is $p$-connected, and a piecewiselinear map $f_{k}: Q_{k} \rightarrow U_{(n-k) p}$ such that $\operatorname{dim} S\left(f_{k}\right) \leq n-k-1$ and $R \subset f_{k}\left(Q_{k}\right)$.

For $k=1$, let $Q_{1}=R \cup C\left(R^{p}\right)$ where $C\left(R^{p}\right)$ is the union of the cones over $p$-skeleta of components of $R$. Define $f_{1}: Q_{1} \rightarrow U_{(n-1) p}$ by extending the identity on $R$ over successive skeleta of $Q_{1}$ using the fact that singular spheres in $U_{i+1}$ are null-homotopic in $U_{i}$ and general position. Then

$$
\begin{aligned}
\operatorname{dim} S\left(f_{1}\right) & \leq \max \left\{\operatorname{dim} S\left(f_{1} \mid Q_{1}-R\right), \operatorname{dim}\left(f_{1}\left(Q_{1}-R\right) \cap R\right)\right\} \\
& \leq n-2
\end{aligned}
$$


Now assume the claim for $1 \leq k-1 \leq n-1$. Let $R_{k-1}=f_{k-1}\left(Q_{k-1}\right)$ and construct $Q_{k}$ by applying Lemma 2 and Lemma 3 with $r=n-k$ to each component of $R_{k-1}$. Construct $f_{k}$ by extending the identity on $R_{k-1}$ over successive skeleta of $Q_{k}$ as before. Then

$$
\operatorname{dim} S\left(f_{k}\right) \leq(n-k+3)+q-m \leq n-k-1
$$

since $q \leq m-4$. By induction $R \subset R_{k-1}$ and by construction $R_{k-1} \subset f_{k}\left(Q_{k}\right)$, so $R \subset f_{k}\left(Q_{k}\right)$.

Therefore, there is an embedding $f_{n}: Q_{n} \rightarrow U_{0}$ where $Q_{n}$ is a q-dimensional polyhedron each component of which is p-connected. Since $R \subset f_{n}\left(Q_{n}\right)$ and a regular neighborhood of $R$ contains $X$, there is a regular neighborhood of $f_{n}\left(Q_{n}\right)$ containing $X$ which is the neighborhood of $X$ desired to complete the proof.

4. Proofs of the remaining cases. For case (c), essentially the same proof works, but an improved version of Lemma 3 is needed because of the lack of codimension. The following lemma satisfies this need. Its proof is omitted since it is quite similar to the last part of the proof of Lemma 3.

Lemma 4. Let $R$ be a connected $q$-dimensional polybedron, $q \geq 2$. If $\pi_{1}(R)$ is a finitely generated free group and $H_{2}(R)=0$, then there exists a 2-connected $q \cdot$ dimensional polybedron $Q$ containing $R$ sucb that $Q-R$ is at most 2-dimensional.

To prove the theorem in case (c), choose $U_{4} \subset U_{3} \subset U_{2} \subset U_{1} \subset U_{0}$ as before. Let $R_{1}$ be the union of the spines for components of $U_{4}$ and $Q_{1}=R_{1} \cup C\left(R_{1}^{2}\right)$ where $C\left(R_{1}^{2}\right)$ is the union of the cones over the 2-skeleta of components of $R_{1}$. Construct $f_{1}: Q_{1} \rightarrow U_{2}$ with $\operatorname{dim} S\left(f_{1}\right) \leq 0$ and let $R_{2}=f_{1}\left(Q_{1}\right)$. This much is the same as in case (b); now, though, we want to apply Lemma 4 to each component $R$ of $R_{2}$. By Lemma 2, $H_{2}(R)=0$. We have $\pi_{1}(R)$ is finitely generated and free since $R$ is obtained from a 2 -connected polyhedron by identifying finitely many pairs of points. Hence, Lemma 4 yields a q-dimensional polyhedron $Q_{2}$ each component of which is 2 -connected such that $\operatorname{dim}\left(Q_{2}-R_{2}\right) \leq 2$. We now extend the inclusion $R_{2} \subset U_{2}$ to a map $f_{2}: Q_{2} \rightarrow U_{0}$ (using $U V^{2}$ as before) such that $\operatorname{dim} S\left(f_{2}\right) \leq 2+q-m=-1$. Then $f_{2}$ is an embedding and there is a regular neighborhood of $f_{2}\left(Q_{2}\right)$ which satisfies the conclusion of the theorem.

In case (d), each component of $X$ is cell-like by a result of Lacher [16, Corollary 4.4 (2)]. Therefore, $X$ has an arbitrarily small neighborhood each component of which is an $m$-cell by [19] and [22].

5. Remarks and similar theorems. For a set which is cellular, we know about the boundary of its cell neighborhoods. Similarly, for a compactum which is semicellular, we remark that if $N$ is a $p$-connected $m$-manifold that has a $q$-spine, 
then $\mathrm{Bd} N$ is $n$-connected where $n=\min \{p, m-q-2\}$ by a simple general position argument.

Because of a result of J. Hollingsworth and R. B. Sher [12], we observe that in Theorem 1 if $\bar{H}^{i}(X)=0$ for $i \geq q+1$ and $q=2$ or 3 , then we need not assume $M$ to be piecewise-linear, since in this situation $X$ will have a piecewise-linear neighborhood regardless. Similarly, if each component of $X$ has $U V^{p}$ and $p \geq 4$, some theorems of Lacher [16, Theorem 3.2] again allow us to prove that $\bar{H}^{4}\left(X ; Z_{2}\right)=0$ and the hypothesis that $M$ be piecewise-linear is unnecessary.

We also mention that the hypothesis that each component of $X$ have $U V^{1}$ in case (a) can be replaced by the weaker hypothesis that there exists a neighborhood $V$ of $X$ such that every loop in $V$ is null-homotopic in $M$. This hypothesis together with $C C$ implies $U V^{1}$.

We now give two more theorems which are variations of Theorem 1. The proofs are not new although the statements may be.

Theorem 5. Let $X$ be a compact proper subset of the interior of a piecewise. linear m.manifold $M$ with $m \geq 4$. If eacb component of $X$ bas $U V^{1}$ and given any (connected) manifold $U$ in $M$ with $\mathrm{Bd} U \cap X=\varnothing, U-(X \cap U)$ is connected, then $X$ bas an arbitrarily small, compact neighborbood eacb component of which is a polybedral m-manifold that bas an $(m-2)$-spine.

Proof. (Compare this to Theorem 2 of [19].) Let $N_{1}$ and $N_{2}$ be arbitrarily small compact neighborhoods of $X$ each component of which is a polyhedral $m$ manifold such that $N_{2} \subset$ Int $N_{1}$ and every loop in $N_{2}$ is null-homotopic in Int $N_{1}$. Let $M_{1}$ be a component of $N_{1}$ and $M_{2}=M_{1} \cap N_{2}$. As in the proof of Theorem 1, case (a), we show that the pair (Int $M_{1}$, Int $M_{1}-X$ ) is 1 -connected. It is 0 -connected since Int $M_{1}$ is. Now let

$$
f:(I, \mathrm{Bd} I) \rightarrow\left(\text { Int } M_{1}, \text { Int } M_{1}-X\right)
$$

represent an element of $\pi_{1}$ (Int $M_{1}$, Int $\left.M_{1}-X\right)$. Cover $f^{-1}(X)$ by a finite number of disjoint subintervals $J_{i}$ such that $f\left(J_{i}\right) \subset M_{2}$ and $f\left(B d J_{i}\right) \subset M_{2}-X$. The points $f\left(\mathrm{Bd} J_{i}\right)$ can be joined by a path $g_{i}: J_{i} \rightarrow M_{2}-X$ by hypothesis. Each loop $g_{i} \cdot\left(f \mid J_{i}\right)^{-1}$ is null-homotopic in Int $M_{1}$, so $f$ is homotopic in Int $M_{1}$ to a path in Int $M_{1}-X$, namely the path obtained by replacing $f \mid J_{i}$ by $g_{i}$ for each subinterval $J_{i}$. The proof is finished now exactly as it was in Theorem 1, case (a).

Theorem 6. Let $X=f(Q)$ be a topological embedding of a compact piecewise. linear q-manifold $Q$ in the interior of a piecewise-linear m-manifold $M$. If $X$ bas CC, $X$ is 1-connected and $q \leq m-3, m \geq 6$, then $X$ bas an arbitrarily small, compact polyedral manifold neighborbood which collapses to a piecewise-linear embedding $g(Q)$. 
Proof. Given a neighborhood $U$ of $X$, there is a compact polyhedral neighborhood $V$ of $X$ such that $V \subset$ Int $U, V$ is a manifold, $V$ deformation retracts to $X$ and $\mathrm{Bd} V$ is simply connected [9, Theorem 4.6]. The topological embedding $f$ of $Q$ can be approximated by a piecewise-linear embedding $g$ of $Q$ into $V$ so close that $f \simeq g$ in $V$ [21]. Since $f: Q \rightarrow V$ is a homotopy equivalence, so is $g: Q \rightarrow V$. Since $V$ and $\operatorname{Bd} V$ are simply connected $V$ collapses to $g(Q)$ [18, Theorem 10.3] and the proof is complete.

This theorem points out that Theorem 1 is only "best possible" when little is known about $X$. When more is known about $X$, it should be possible to obtain more precise information about the spine. Theorem 6 is one step in this direction.

Attempts to extend Theorem 1 to include the case $p=0$ are probably pointless since, for $\operatorname{dim} X \leq m-2$, if $X$ has $C C$, then the components of $X$ have $U V^{1}$, and for $\operatorname{dim} X=m-1$, then $R P^{m-1} \subset R P^{m}$ (m-odd) provides one counterexample. ( $R P^{k}$ denotes $k$-dimensional projective space.) On the other hand, there is hope of extending the result in the direction of requiring less codimension: that is $q=m-2$ or $q=m-3, m \geq 4$. For $p=1, m \geq 5$ this has been done (Theorem 1 (a)) and for $p=2$, this has been partly done (Theorem 1 (c)). Notice that getting neighborhoods with a $q$-spine is not the problem (Theorem 1 (a) and Theorem 5); the difficulty is to obtain the p-connectivity. Extending Theorem 1 (d) to include $m=3$ and $m=4$ is hindered by the Poincaré conjecture. One still obtains celllikeness for the components of $X$ and if $m=3$ and some neighborhood of $X$ embeds in $E^{3}$, the conclusion still holds [19].

6. Extending $U V^{p}$ decompositions. We now apply the semicellularity criterion (Theorem 1) to the problem of extending decompositions. The standard terminology for decompositions will be used without definition; refer to [11] or [14]. A decomposition is a $U V^{p}$-decomposition if each element is a compact set with property $U V^{p}$. A $U V^{0}$-decomposition is more frequently known as a monotone decomposition.

The theorems are stated in terms of maps rather than decompositions for convenience. A map $f: M \rightarrow N$ between manifolds is a proper map if $f$ is closed and $f^{-1}(y)$ is compact for every $y \in N$. A proper map is a $U V^{p}$-map provided each point inverse has property $U V^{p}$. A proper, surjective, $U V^{p}$-map induces an upper semicontinuous, $U V^{p}$ decomposition $G_{f}$ defined by $G_{f}=\left\{f^{-1}(y) \mid y \in N\right\}$. This correspondence allows us to state as corollaries analogous results in terms of decompositions.

Theorem 7. Let $X$ be a compact subset of Int $M$ where $M$ is a compact, $(p+1)$-connected, piecewise-linear n-manifold, $\mathrm{Bd} M$ is nonempty and $p$-connected, $p \geq 1$ and $n \geq 6$. If $X$ bas $C C$, each component of $X$ bas $U V^{p}$ and $\bar{H}^{i}(X)=0$ for $i \geq n-p-1$, then there is a surjective, $U V^{p_{-m a p}} f: M \rightarrow B^{n}$ such that 
$f(B d M)=S^{n-1}, f($ Int $M)=$ Int $B^{n}, f(X)$ is a tame zero-dimensional set and for each $y \in N, f^{-1}(y)$ is a point, a component of $X$ or an $(n-p-2)$-dimensional poly. bedron disjoint from $X$.

The following two lemmas will be needed in the proof of Theorem 7 and in subsequent theorems.

Lemma 8. Let $W$ be a compact piecewise-linear m-manifold such that both $W$ and $\mathrm{Bd} W$ are q-connected $(\mathrm{Bd} W \neq 0$ ) where $2 q+3 \leq m$. Then the following statements are true.

(i) W bas a q-connected, ( $m-q-1)-s$ pine.

(ii) If $H_{q+1}(W, \mathrm{Bd} W)=0$ and $q \geq 1, m \geq 6$, then $W$ bas a q-connected, (m-q-2)-spine.

(iii) If $W$ is $(q+1)$-connected and $q \geq 1, m \geq 6$, then $W$ bas $a(q+1)$-connected, $(m-q-2)$-spine.

The proof is a standard handle cancellation argument and is implicit in most proofs of the high dimensional Poincaré theorem or b-cobordism theorem; see [13], [18]. The details were written out in [8]. Consequently, we omit the proof here.

Lemma 9. Let $W$ be a compact, piecewise-linear m-manifold which bas nonempty, connected boundary and a p-connected $q$-spine $K$ for some $p$ and $q$. Let $f: \mathrm{Bd} W \rightarrow S^{m-1}$ be a surjective $U V^{p_{-m a p}}$ such that each nondegenerate point. inverse is a p-connected polybedron of dimension $\leq q$. Then $f$ extends to $F: W \rightarrow B^{m}$ such that $F$ is a UV $V^{p}$-map, each nondegenerate point-inverse of $F$ is a p-connected polybedron of dimension $\leq q$, and $F(\operatorname{Int} W)=\operatorname{Int} B^{m}$.

Proof. There is a piecewise linear homeomorphism

$$
b:(W-K, \mathrm{Bd} W) \rightarrow(\mathrm{Bd} W \times(0,1], \mathrm{Bd} W \times\{1\}) .
$$

Let $b_{1}$ denote $b$ followed by projection onto $\mathrm{Bd} W$ and $b_{2}^{\prime}$ denote $b$ followed by projection onto $(0,1]$. Consider $B^{m}-\{0\}$ to have spherical coordinates:

$$
B^{m}-\{0\}=\left\{(s, t) \mid s \in S^{m-1}, t \in(0,1]\right\} \text {. }
$$

Define

$$
F(x)=\left\{\begin{array}{l}
0, \text { if } x \in K, \\
\left(f b_{1}(x), b_{2}(x)\right), \quad \text { if } x \in W-K .
\end{array}\right.
$$

Clearly this $F$ satisfies the conclusion of the lemma.

Remark. If / has finitely many nondegenerate point-inverses, say $k$, then the image of the nondegenerate point-inverses of $F$ is a $k$-frame, or $k$-od, with its branch point the image of $K$.

The following proof of Theorem 7 owes much to Bing's proof of Theorem 7.3 in [5] and Bean's proof of Theorem 2 in [4]. 
Proof. By Theorem 1(a), (b) and (d), $X$ is the intersection of a sequence of compact, polyhedral manifolds $M_{i}$ where $M_{1}=M$, and for each $i, M_{i+1} \subset \operatorname{Int} M_{i}$ and each component $M_{i}$ is a $p$-connected $n$-manifold that has an $(n-p-2)$-spine. If $2 p+2 \geq n$, notice that for all $i$ each component of $M_{i}$ is an $n$-cell. This is given by Theorem 1(d) for $i \geq 2$ and by an argument using duality and the universal coefficient theorem for $M_{1}=M$. In $B^{n}$ choose a sequence of finite collections of polyhedral $n$-cells $C_{i}$ such that there is a one-to-one correspondence between the components of $M_{i}$ and the cells of $C_{i}$ which commutes with the inclusion relations between components, and such that the intersection of the $C_{i}$ 's is a tame zero-dimensional set. Take $C_{1}=B^{n}$.

The desired map $/$ will be defined inductively. Specifically, we claim that for each $k$

$$
f \mid M-\text { Int } M_{k}: M-\text { Int } M_{k} \rightarrow B^{n}-\text { Int } C_{k}
$$

can be defined such that there is at most one nondegenerate point-inverse on each component of $\mathrm{Bd} M_{k}$ in addition to the relevant properties listed in the conclusion. For $2 p+2 \geq n$, and for all $k$, this map can be taken to be a homeomorphism. Hence, throughout the following induction argument we assume $2 p+3 \leq n$.

For $k=1$, we define $f \mid \mathrm{Bd} M: \mathrm{Bd} M \rightarrow S^{n-1}$ as follows. If $2 p+4 \leq n$, define $f$ first on an $(n-1)$-ball in $\mathrm{Bd} M$ to be a homeomorphism onto an $(n-1)$-ball in $S^{n-1}$. Then apply Lemma 8(i) with $q=p$ and $m=n-1$ to the closure of the complement of this ball in $\mathrm{Bd} M$ and define $f \mid \mathrm{Bd} M$ by Lemma 9 . For $2 p+3=n$ use the same approach except to substitute Lemma 8(iii) with $q=p-1$ and $m=n-1$. Notice that if $2 p+3=n$ and $n \geq 6$, then $n \geq 7$ and $p \geq 2$.

Now assume $f \mid M-$ Int $M_{k}$ has been defined and $k \geq 1$. First choose a (minimal) collection of disjoint tubes (relative regular neighborhoods of arcs) joining $\mathrm{Bd} M_{k+1}$ to $\mathrm{Bd} M_{k}$ in $M_{k}$ - Int $M_{k+1}$ which miss the nondegenerate pointinverses in Bd $M_{k}$. Let $T_{k}$ denote the union of these tubes and define $f$ on $T_{k}$ to be a homeomorphism onto a union of corresponding tubes in $C_{k}-\operatorname{Int} C_{k+1}$.

Secondly, let

$$
U_{k+1}=\mathrm{Cl}\left[\mathrm{Bd} M_{k+1}-\left(T_{k} \cap \mathrm{Bd} M_{k+1}\right)\right] \text {. }
$$

Note that $T_{k} \cap \mathrm{Bd} M_{k+1}$ is a collection of $(m-1)$-cells. Hence, each component of $\mathrm{Bd} U_{k+1}$ is $p$-connected since $p \leq m-3$. Also, since each component of $M_{k+1}$ is $p$-connected and has an $(n-p-2)$-spine, a general position argument allows us to conclude that each component of $B d M_{k+1}$ and, hence, of $U_{k+1}$ is $p$-connected. Therefore, $U_{k+1}$ has an $(n-p-2)$-spine each component of which is $p$-connected by Lemma $8(\mathrm{i})$ if $2 p+4 \leq n$ and by Lemma 8 (iii) if $2 p+3=n$. Now define $f$ on $U_{k+1}$ by Lemma 9 . 
Thirdly, let

$$
N_{k}=\mathrm{Cl}\left[M_{k}-\left(T_{k} \cup M_{k+1}\right)\right]
$$

and

$$
D_{k}=\mathrm{Cl}\left[C_{k}-\left(f\left(T_{k}\right) \cup C_{k+1}\right)\right] \text {. }
$$

The same sort of general position argument as above allows us to conclude that each component of both $N_{k}$ and $\mathrm{Bd} N_{k}$ is p-connected. Furthermore, $H_{p+1}\left(V_{k}, \mathrm{Bd} N_{k}\right)=0$ by excision since $H_{p}\left(N_{k}\right)=0$ and $H_{p+1}(M)=0$. Therefore, by Lemma $8(\mathrm{ii}), N_{k}$ has an $(n-p-2)$-spine each component of which is $p$-connected. Again define $f$ on $N_{k}$ by Lemma 9. Thus, we have defined $f$ on all of $M_{k}$ - Int $M_{k+1}$, so $/ \mid M$ - Int $M_{k+1}: M$ - Int $M_{k+1} \rightarrow B^{n}-$ Int $C_{k+1}$ is defined. We have completed the inductive step.

Therefore, we can define

$$
f \mid M-\bigcap_{k=1}^{\infty} M_{k}: M-\bigcap_{k=1}^{\infty} M_{k} \rightarrow B^{n}-\bigcap_{k=1}^{\infty} C_{k} .
$$

Each component $Y$ of $X$ is uniquely determined by a sequence of components one from each $M_{k}$ and the corresponding cells of $C_{k}$ intersect in a point which will be, by definition, the image of $\%$.

This completes the definition of the map $f$ and it has the desired properties by construction so the proof is finished.

Remark. Because of the remark following the proof of Lemma 9, we can observe that the image of the nondegenerate point-inverses of $f$ is an infinite tree which intersects each component of $\mathrm{Bd} C_{k}$ at most once and ends on $f(X)$.

Remark. Assuming that $M$ is $p$-connected but not $(p+1)$-connected, the proof gives the same conclusion except that a countable number of $(n-p-1)$ dimensional polyhedra may occur as nondegenerate point-inverses. These are the spines of the manifolds $N_{k}$ in the proof. This happens because the application of Lemma $8(\mathrm{ii})$ requires $H_{p+1}\left(N_{k}, \mathrm{Bd} N_{k}\right)=0$ which results from $H_{p+1}(M)=0$. No further improvements can be made in this direction since a $U V^{p}$-map induces isomorphisms of homotopy groups $\pi_{i}$ for $i \leq p,[2$, Theorem 5.1], [15, Theorem 3.1].

Similarly, in the case $n=5$, the proof yields the same conclusion except that the dimension of all the added nondegenerate point-inverses may be $n-p-$ $1=3$. This occurs because neither 1-connected 4-manifolds nor l-connected 5-manifolds necessarily have 2-spines, but they do have 3-spines trivially and by Lemma 8(i), respectively.

7. Extending monotone decompositions in high dimensions. In this section we prove the $p=0$ analogue of Theorem 7. The proof is similar in some respects to 
R. H. Bing's proof of this theorem in dimension 3, but the exact analogue of his proof fails.

Theorem 10. Let $X$ be a compact subset of Int $M$ where $M$ is a compact, piecewise-linear, connected n-manifold with nonempty connected boundary, $n \geq 3$. If $\bar{H}^{i}(X)=0$ for $i \geq n-1$, then there is a surjective, monotone map $f: M \rightarrow B^{n}$ sucb that $f(\mathrm{Bd} M)=S^{n-1}, f($ Int $M)=$ Int $B^{n}, f(X)$ is a tame zero-dimensional set, and each point-inverse of $f$ is a point, a component of $X$, or an $(n-2)$-dimen. sional polybedron disjoint from $X$.

The proof is similar to that of Theorem 7, but we do not get the $(n-2)$-spine from Lemma 8 whose complement is a product with $(0,1]$. We can get a "layering" of sorts in the next lemma, however.

For that we need some more definitions. A pinched manifold is the identification space obtained from a manifold by identifying a single pair of points. The image of this pair is called the pinch point. A p-bandle attached to a piecewise linear $n$-manifold $W$ is a copy of $B^{p} \times B^{n-p}$ attached to $B d W$ by means of a homeomorphism on $S^{p-1} \times B^{n-p}$. Refer to [13] or [18] for additional definitions or background on handles.

Lemma 11. Let $W$ be a compact, connected, piecewise-linear n-manifold with connected, nonempty boundary and $n \geq 3$. Then there is a monotone, piecewise. linear map $g: W \rightarrow[0,1]$ satisfying the following properties:

(i) $g^{-1}(1)=\mathrm{Bd} W$;

(ii) $g^{-1}(0)$ is an $(n-2)$-dimensional polybedron;

(iii) there is a finite collection $1 / 2<t_{1}<t_{2}<\cdots<t_{k}<1$ where $g^{-1}\left(t_{i}\right)$ is a pinched manifold;

(iv) if $J=\left(0, t_{1}\right),\left(t_{i}, t_{i+1}\right)$, or $\left(t_{k}, 1\right]$ and $t \in J$, then $g^{-1}(t)$ is an $(n-1)$. manifold and $g^{-1}(J) \cong g^{-1}(t) \times J$ by a bomeomorphism which takes $g^{-1}(s)$ to $\mathrm{g}^{-1}(t) \times\{s\}$ for eacb $s \in J$; and

(v) there is an $\epsilon>0$ and for each $i$ a projection $p_{i}: g^{-1}\left(\left[t_{i}-\epsilon, t_{i}+\epsilon\right]\right) \rightarrow$ $g^{-1}\left(t_{i}\right)$ such that for $t \in\left[t_{i}-\epsilon, t_{i}+\epsilon\right], p_{i} \mid g^{-1}(t)$ is one-to-one except on the pinch point and $\left(p_{i} \mid g^{-1}(t)\right)^{-1}$ of the pinch point is bomeomorpbic to $S^{n-2}$ for $t<t_{i}$ and is two points $t>t_{i}$.

Proof. Since $W$ and $\mathrm{Bd} W$ are connected, $W$ can be obtained from a collar $C$ of $\mathrm{Bd} W$ by attaching handles in increasing order none of which are 0 -handles. Let $U$ be $C$ union all of the 1-handles and $V=\mathrm{Cl}(W-U)$. Then $V$ has a handle decomposition with handles of dimension $n-2$ at most which is obtained by "reversing the handles". Consider each $p$-handle $b^{p}=B^{p} \times B^{n-p}$ as $k^{n-p}=$ $B^{n-p} \times B^{p}$. The order of attachment is also reversed and since $B d W \cap V=\varnothing$, 
each $k^{n-p}$ is attached as an $(n-p)$-handle. Then $V$ has an $(n-2)$-spine $K$. Let $V-K$ be identified with $\mathrm{Bd} V \times(0,1 / 2]$ and define $g$ on $V-K$ by $g(b, t)=t$ for $(b, t) \in \operatorname{Bd} V \times(0,1 / 2]$. Define $g$ in $K$ by $g(K)=0$.

Now let $C$ be identified with $B d W \times[1 / 2,1]$ such that $B d W$ is $B d W \times\{1\}$. The attaching tube of each 1-handle is a copy of $S^{0} \times B^{n-1}$ lying in $\mathrm{Bd} W \times\{1 / 2\}$. For each 1-handle $b_{i}$ in $U$, let $D_{i}$ and $E_{i}$ be caps in $C$ over the two components of the attaching tube of $b_{i}$ defined as the cone over each component to the point in $\mathrm{Bd} W \times\{3 / 4\}$ corresponding to the image of $0 \in B^{n-1}$. Define $g$ on $C-\left(\cup\left(D_{i} \cup E_{i}\right)\right)$ by $g(h, t)=t$ where $(b, t) \in \mathrm{Bd} W \times[1 / 2,1]$. In each one-handle $b_{i}$ let $x_{i}$ be the image of $(0,0) \in B^{1} \times B^{n-1}$. Choose $t_{i} \in(1 / 2,3 / 4)$, different for each $i$, and define $g\left(x_{i}\right)=t_{i}$. Extend $g$ to $b_{i} \cup D_{i} \cup E_{i}$ by coning. This completes the definition of $g$. The properties listed in the conclusion can now be easily checked noting that each layer $g^{-1}(t)(t \neq 0)$ is obtained from BdW by removing pairs of $(n-1)$-cells and replacing each pair by another pair of $(n-1)$ cells, an annulus $B^{1} \times S^{n-2}$, or (in finitely many cases) the cone over two copies of $S^{n-2}$.

Next we need a lemma corresponding to Lemma 9 for the type of layering just obtained.

Lemma 12. Let $W$ be a compact, connected, piecewise-linear n-manifold, $n \geq 3$, with connected, nonempty boundary. If $f: \mathrm{Bd} W \rightarrow S^{n-1}$ is a monotone map sucb that the nondegenerate point-inverses of $f$ form a finite collection of $(n-2)$ dimensional polybedra, then $f$ extends to a monotone map $F: W \rightarrow B^{n}$ such that eacb nondegenerate point-inverse of $F$ is an $(n-2)$-dimensional polybedron.

Proof. Let $g: W \rightarrow[0,1]$ be the map given by Lemma 11 . Assume the intervals $\left[t_{i}-\epsilon, t_{i}+\epsilon\right]$ are mutually disjoint. There is a map

$$
P_{k}: g^{-1}(1) \times\left[t_{k}, 1\right] \rightarrow g^{-1}\left(\left[t_{k}, 1\right]\right)
$$

such that $P_{k} \mid g^{-1}(1) \times\{s\}$ is the homeomorphism given in (v) if $s \neq t_{k}$, and $P_{k}\left|g^{-1}(1) \times\left\{t_{k}\right\}=p_{k}\right| g^{-1}(1)$. Choose a tame arc $A$ in Bd $W$ such that $A$ contains the pair of points $\left(p_{k} \mid g^{-1}(1)\right)^{-1}$ of the pinch point of $g^{-1}\left(t_{k}\right)$, and $A$ intersects the nondegenerate point-inverses in exactly an endpoint. (Assume this pair of points is disjoint from the nondegenerate point-inverses to avoid trivial case-taking.) Then $f(A)$ is an arc in $S^{n-1}$, and there is a pseudo-isotopy $b$ : $S^{n-1} \times I \rightarrow S^{n-1} \times I$ shrinking $f(A)$ to a point. Now define $F \mid g^{-1}\left(\left[t_{k}, 1\right]\right)$ by $F(x)=b\left(f \times \mathrm{id}\left(P_{i}^{-1}(x)\right)\right)$. This is well defined since $P_{i}$ is one-to-one everywhere except onto the pinch point of $g^{-1}\left(t_{k}\right)$, and $b$ was chosen to correct that. Then define $F$ on $g^{-1}\left(\left[t_{k}-\epsilon, t_{k}\right]\right)$ by $F(x)=F\left(p_{k}(x)\right)$. Next define $F$ successively on each $g^{-1}\left(\left[t_{i}-\epsilon, t_{i+1}-\epsilon\right]\right)$ in the same way as above. Finally define $F$ on $\mathrm{g}^{-1}\left(\left[0, t_{1}-\epsilon\right]\right)$ by Lemma 9 , and the proof is complete. 
Proof of Theorem 10. Follow the same steps as in the proof of Theorem 7 except to use Lemma 12 instead of Lemmas 8 and 9 when it comes to defining the map $f$ on the manifold $N_{k}=\mathrm{Cl}\left[M_{k}-\left(T_{k} \cup M_{k+1}\right)\right]$. Notice that on Bd $M$ and on $U_{k+1}=\mathrm{Cl}\left[\mathrm{Bd} M_{k+1}-\left(T_{k} \cap \mathrm{Bd} M_{k+1}\right)\right]$ there is no problem since an $n-1$ manifold with boundary has an $(n-2)$-spine trivially.

Remark. Theorems similar to Theorems 7 and 10 can be proven in the case $\mathrm{Bd} M=\varnothing$. Simply map a ball disjoint from $X$ homeomorphically onto $\mathrm{Cl}\left(S^{n}-B^{n}\right)$ as the first step in the induction; the rest is the same. Of course, here the range is $S^{n}$, not $B^{n}$; and one should add $X \neq M$ to the hypothesis. We state this version in terms of decompositions to illustrate the relationship between the two pointsof-view.

Corollary 13. Let $M$ be a compact, $(p+1)$-connected, piecewise-linear nmanifold with $\mathrm{Bd} M$ empty, $n \geq 3, p \geq 0$. Let $G$ be an upper semicontinuous $U V^{p}$ decomposition of $M$ whose nondegenerate elements are the components of a compact set $X$. Assume $\bar{H}^{i}(X)=0$ for $i \geq n-p-1$; and if $p \geq 1$ assume $X$ bas $C C$, and $n \geq 6$. Then there is an upper semicontinuous $U V^{p}$ decomposition $H$ of $M$ which extends $G$ (in the sense that eacb nondegenerate element of $G$ is an element of $H$ ), sucb that $M / H \cong S^{n}$, and the nondegenerate elements of $H$ wbich are not in $G$ are $(n-p-2)$-dimensional polybedra.

This is Bing's theorem [5, Theorem 7.3] in the case that $p=0$ and $n=3$ since the condition that $\bar{H}^{i}(X)=0$ for $i \geq n-1$ implies the non-locally-separating condition of Bing.

8. Semicellularity criteria for maps. We now give some criteria for the pointinverses of a map between manifolds to be $(p, q)$-semicellular. There are fewer conditions needed than for an arbitrary set. Lacher first noticed this in relation to cellularity. The following theorem includes his result [16, Theorem 5.4] for completeness.

Theorem 14. Let $f: M \rightarrow N$ be a proper, surjective, UV $V^{p}$ map between manifolds without boundary where $M$ bas dimension $m \geq 5, N$ bas dimension $n \geq 3$, and $p \geq 1$. Then the following may be concluded:

(i) If $m \leq 2 p+1$, then each point-inverse is cellular:

(ii) If $m=2 p+2$ and $H_{p_{0}+1}(M)=0$, then each point-inverse is cellular.

(iii) If $m \geq 2 p+2$, then $f^{-1}(y)$ bas a compact, p-connected, polybedral maniifold neigbborbood which bas an $(m-p-1)$-spine for all $y \in N$.

(iv) If $m \geq 2 p+2$, then $f^{-1}(y)$ bas a compact, $p$-connected, polybedral manifold neighborbood whicb bas an $(m-p-2)$-spine for all but a locally finite subset of points $y \in N$. 
(v) If $m \geq 2 p+3$ and $H_{p+1}(M)=0$, then $f^{-1}(y)$ bas a compact, $p$-connected, polybedral manifold neighborbood which has an $(m-p-2)$-spine for every $y \in N$.

Before we prove Theorem 14, we need the following lemmas.

Lemma 15. Let $f: M \rightarrow N$ be a proper, surjective, $U V^{1}$-map between manifolds without boundary where $N$ bas dimension $n \geq 3$. If $Y$ is a compact, zerodimensional set in $N$ which is contained in an $n$-ball and is tame there, then $f^{-1}(Y)$ bas $C C$.

Proof. Let $X=f^{-1}(Y)$. Given an open set $U$ containing $X$ choose a finite collection of open balls $B$ in $f(U)$ containing $Y$, and let $V=f^{-1}(B)$. On each component of $V-X, f$ is a $U V^{1}$-map so $f$ induces isomorphisms on the fundamental groups by [2, Theorem 5.1], [15, Theorem 3.1]. Since $n \geq 3, B-Y$ is simply connected. Hence, $X$ has $C C$.

Lemma 16. Let $f: M \rightarrow N$ be a proper, surjective, $U V^{p}$-map between manifolds without boundary webre $M$ bas dimension $m, N$ bas dimension $n$ and $1 \leq p \leq n-2$. Then:

(i) $\bar{H}^{i}\left(f^{-1}(y)\right)=0$ for $i \geq m-p$ for every $y \in N$;

(ii) $\bar{H}^{m-p-1}\left(f^{-1}(y)\right)=0$ for all but a locally finite set of points $y \in N$; and

(iii) if $H_{p+1}(M)=0$, then $\bar{H}^{m-p-1}\left(f^{-1}(y)\right)=0$ for every $y \in N$.

Proof. Statements (i) and (ii) are proven in [17]; see Theorems 1.3, 2.3 and $\$ 4$. To prove (iii) consider the commutative diagram:

$$
\begin{aligned}
H_{p+1}(M) \rightarrow H_{p+1}(M, M-X) & \rightarrow H_{p}(M-X) \stackrel{a}{\rightarrow} H_{p}(M) \\
& \left.\downarrow(f \mid M-X)_{*}\right|^{\mid} f_{*} \\
& H_{p}(B-\{y\}) \rightarrow H_{p}(B) \oplus H_{p}(N-\{y\}) \stackrel{\beta}{\longrightarrow} H_{p}(N)
\end{aligned}
$$

In this diagram $B$ denotes a ball containing $y$ and $X=f^{-1}(y)$. The top line is part of the exact sequence of the pair $(M, M-X)$ and the bottom line is part of the Mayer-Vietoris sequence for $N=B \cup(N-\{y\})$. Since $f$ is a $U V^{p}$-map $(f \mid M-X)_{*}$ and $f_{*}$ are isomorphisms [16, Lemma 5.1]. Therefore, $a$ is an injection since $H_{p}(B-\{y\})=H_{p}(B)=0$. By hypothesis $H_{p+1}(M)=0$, so by exactness $H_{p+1}(M, M-X)=0$. This completes the proof since by duality [23, Theorem 6.2.17] $H_{p+1}^{p+1}(M, M-X) \cong \bar{H}^{m-p-1}(X)$. (Note: An orientable neighborhood can be obtained since $X$ has $U V^{1}$ as in $\$ 2$. Use this if $M$ is nonorientable.)

Proof of Theorem 14. Lacher [16, Corollary 4.4(2)] has shown that if a compact set $X$ has property $U V^{p}$ and $\bar{H}^{i}(X)=0$ for $i \geq p+1$, then $X$ has property $U V^{\infty}$. Thus, the cellularity criterion of McMillan [19] yields (i) and (ii), and the semicellularity criterion, Theorem 1, yields (iii), (iv) and (v). 
Remark. Lemmas 15 and 16 also prove a converse of Theorem 7. For this we need the full strength of Lemma 15, rather than the version where $Y=\{y\}$ used in the proof of Theorem 14. The corresponding strengthening of Lemma 16 is not needed since $\bar{H}^{i}\left(f^{-1}(Y)\right)=0$ if and only if $\bar{H}^{i}\left(\digamma^{-1}(y)\right)=0$ for every $y \in Y$ where $\boldsymbol{Y}$ is a compact, zero-dimensional set.

Lacher [16] has proven that Theorem 14 (i) and (ii) are true assuming only that $f$ is a $U V^{p-1}$-map and a $p-u v(Z)$-map. The latter means that for each $y \in N$, given an open set $U$ containing $f^{-1}(y)$, there is an open set $V$ with $\digamma^{-1}(y) \subset$ $V \subset U$ such that $i: V \subset U$ induces the zero homomorphism $H_{p}(V ; Z) \rightarrow H_{p}(U ; Z)$. Improving this result slightly, we have the following.

Theorem 17. Let $f: M \rightarrow N$ be a proper, surjective, $U V^{p-1}$ and $p-u v\left(Z_{q}\right)$ map where $M$ bas dimension $m \geq 5, N$ bas dimension $n \geq 3, \mathrm{Bd} M=\mathrm{Bd} N=\varnothing$ and $q$ is a prime integer. If $m=2 p$, or if $m=2 p+1$ and $H_{p}(M)=0$, then $f^{-1}(y)$ is cellular for every $y \in N$.

The following lemma, needed to prove Theorem 17, is a generalization of Theorem 3 of [20], and is proven in the same way. The terminology is due to Stallings [26] and is also defined in [20]. Here we use it only for the statement of Lemma 18 and the proof of Theorem 17.

Lemma 18. Let $M$ be a manifold, $Y$ be a Hausdorff space, and $f: M \rightarrow Y$ be a $U V^{p-1}$ and $p-u v\left(Z_{q}\right)$-map where $p \geq 1$ and $q$ is a prime integer. If there is a $y_{0} \in Y$ such that given an open set $U$ containing $\Gamma^{-1}\left(y_{0}\right)$, there is an open set $V$ with $\Gamma^{-1}\left(y_{0}\right) \subset V \subset U$ such that each singular p-sphere in $V$ projects under $f$ to a null-bomotopic p-sphere in $f(U)$, then $f^{-1}\left(y_{0}\right)$ bas an $(\omega, q)$-trivial $\pi_{p}$-shape.

Proof of Theorem 17. Let $y \in N$. Given any neighborhood $U$ of $f^{-1}(y)$ in $M$, choose an open $n$-ball $B$ in $f(U)$ containing $y$. Let $V=f^{-1}(B)$. Then any singular $p$-sphere in $V$ projects under $f$ to a null-homotopic singular $p$-sphere in $f(U)$. By Lemma 18, $f^{-1}(y)$ has an $(\omega, q)$-trivial $\pi_{p}$-shape. To prove that $f^{-1}(y)$ has property $U V^{p}$, let $U^{\prime}$ be any open set containing $f^{-1}(y)$ and choose open sets $V_{1}$ and $V_{2}$ such that $f^{-1}(y) \subset V_{1} \subset V_{2} \subset U ; V_{2}$ is a compact, polyhedral $(p-1)$-connected $m$-manifold that has a $p$-spine; and each singular $p$-sphere in $V_{1}$ represents an element of $F_{\omega}$ of the $q$-central series of $F=\pi_{p}\left(V_{2}\right)$. To obtain $V_{2}$ use Theorem 14(iii) when $m=2 p$ and Theorem 14(v) when $m=2 p+1$. The definition of $(\omega, q)$-trivial $\pi_{p}$-shape yields $V_{1}$. Since $V_{2}$ is $(p-1)$-connected, $F=\pi_{p}\left(V_{2}\right)$ $\simeq H_{p}\left(V_{2}\right)$. But $H_{p}\left(V_{2}\right)$ is a free abelian group since $V_{2}$ has a $p$-spine. Thus, $F$ is a free abelian group and $F_{\omega}=0$ [26]. Therefore, each singular $p$-sphere in $V_{1}$ is null-homotopic in $V_{2} \subset U$, and $f$ has property $U V^{p}$ by definition. Application of Theorem 14(i) completes the proof. 
An example constructed like the dyadic solenoid using regular neighborhoods of $p$-spheres rather than solid tori illustrates that an arbitrary compactum in a manifold can have properties $U V^{p-1}$ and $p-u v\left(Z_{2}\right)$, but not property $U V^{p}$. Theorem 17 says that this cannot happen for point-inverses of a map.

\section{REFERENCES}

1. S. Armentrout, UV properties of compact sets, Trans. Amer. Math. Soc. 143 (1969), 487-498. MR 42 \#451.

2. S. Armentrout and T. M. Price, Decompositions into compact sets with UV properties, Trans. Amer. Math. Soc. 141 (1969), 433-442. MR 39 \#6307.

3. B. J. Ball and R. B. Sher, Extending cell-like maps on manifolds, Notices Amer. Math. Soc. 19 (1972), A466. Abstract \#2T-G76.

4. R. J. Bean, Repairing embeddings and decompositions in $S^{3}$, Duke Math. J. 36 (1969), 379-385. MR 39 \#4820.

5. R. H. Bing, Extending monotone decompositions of 3-manifolds, Trans. Amer. Math. Soc. 149 (1970), 351-369. MR $41 \# 656$.

6. W. Browder, Structures on $M \times R$, Proc. Cambridge Philos. Soc. 61 (1965), 337345. MR 30 \#321.

7. M. Brown, A proof of the generalized Schoenflies theorem, Bull. Amer. Math. Soc. 66 (1960), 74-76. MR $22 \# 8470$ b.

8. D. Coram, Semicellularity of compact subsets of manifolds, Ph. D. thesis, University of Wisconsin, Madison, 1970.

9. P. F. Duvall, Jr., Neighborhoods of 1-connected ANR's in high dimensional piecewise linear manifolds, $\mathrm{Ph}$. D. thesis, University of Georgia, Athens, Ga., 1967.

10. Weakly flat spheres, Michigan Math. J. 16 (1969), 117-124. MR 39 \#604.

11. J. G. Hocking and G. S. Young, Topology, Addison-Wesley, Reading, Mass., 1961. MR 23 \#A2857.

12. John Hollingsworth and R. B. Sher, Triangulating neighborhoods in topological manifolds, General Topology and Appl. 1 (1971), 345-348. MR 45 \#6011.

13. J. F. P. Hudson, Piecewise linear topology, University of Chicago Lecture Notes prepared with the assistance of J. L. Shaneson and J. Lees, Benjamin, New York, 1969. MR $40 \# 2094$. 1136.

14. J. L. Kelley, General topology, Van Nostrand, Princeton, N. J., 1955. MR 16,

15. R. C. Lacher, Cell-like mappings. I, Pacific J. Math. 30 (1969), 717-731. MR 40 \#4941.

16. - Cellularity criterion for maps, Michigan Math. J. 17 (1970), 385-396. MR 43 \#5539.

17. R. C. Lacher and D. R. McMillan, Jr., Partially acyclic mappings between manifolds, Amer. J. Math. 94 (1972), 246-266.

18. W. B. E. Lickorish, Polyhedral handlebody theory, Mimeographed lecture notes, University of Wisconsin, Madison, 1969.

19. D. R. McMillan, Jr., A criterion for cellularity in a manifold, Ann. of Math. (2) 79 (1964), 327-337. MR 28 \#4528.

20. —, Acyclicity in three-manifolds, Bull. Amer. Math. Soc. 76 (1970), 942-964. MR $42 \# 5269$. 
21. R. T. Miller, Approximating codimension 3 embeddings, Proceedings of the Georgia Topology Conference, 1970.

22. R. B. Sher, Defining subsets of manifolds by cells, Bull. Acad. Polon. Sci. Sér. Sci. Math. Astronom. Phys. 17 (1969), 363-365. MR 41 \#1059.

23. E. Spanier, Algebraic topology, McGraw-Hill, New York, 1966. MR 35 \#1007.

24. J. Stallings, The piecewise-linear structure of Euclidean space, Proc. Cambridge Philos. Soc. 58 (1962), 481-488. MR 26 \#6945.

25. - On topologically unknotted spheres, Ann. of Math. (2) 77 (1963), 490503. MR $26 \# 6946$.

26. MR 31 \#232.

DEPARTMENT OF MATHEMATICS, OKLAHOMA STATE UNIVERSITY, STILLVATER, OKLAHOMA 74074 